PUBLIC HEALTH

\title{
Building a sentinel surveillance system for sexually transmitted infections in Germany, 2003
}

\author{
V Bremer, U Marcus, A Hofmann, O Hamouda
}

Sex Transm Infect 2005;81:173-179. doi: 10.1136/sti.2004.009878

See end of article for authors' affiliations

.....................

Correspondence to: Viviane Bremer, MD, $\mathrm{MPH}$, Robert Koch-Institut, Department for Infectious Disease Epidemiology, Seestrasse 10,13353 Berlin, Germany; bremerv@rki.de

Accepted for publication 21 July 2004

\begin{abstract}
Background/objectives: Increases in STIs have been reported from the United States and Europe. Since 2001 , only syphilis and HIV are notifiable in Germany. A sentinel surveillance system has been set up to assess the occurrence and trends of STls and identify risk groups.

Methods: Through the sentinel system data are collected from local health offices (LHO), hospital based STI clinics and private practitioners (dermato-venerology, urology, gynaecology, or HIV). For every newly diagnosed laboratory confirmed infection of HIV, gonorrhoea, chlamydia, syphilis, or trichomoniasis physicians complete a standardised questionnaire regarding diagnosis, source of infection, and demographic information. Patients complete a questionnaire about sexual risk behaviour. The patient form is matched with the diagnosis form using a unique identifier number. Characteristics of sentinel patients were compared with those reported through the HIV and syphilis national notification system.

Results: 58 LHO, 14 hospital based STI clinics, and 160 private practitioners $153.1 \%$ dermatovenerologists) from all federal states participated in the study. $176(75.9 \%)$ sentinel sites are located in cities of >100 000 inhabitants. From 1 March 2003-29 February 2004, a total of 1833 STls have been reported, among them 452 chlamydia, 321 syphilis (10.9\% of notified syphilis), 343 gonorrhoea, 269 HIV (15.7\% of notified HIV). 925 (50.5\%) of the patients were male, the median age was 31 years. Female patients were more often of foreign origin $\left(\chi^{2}\right.$ test; $\left.70.0 \% \vee 26.3 \% ; p<0.001\right)$.

Conclusions: Our sentinel system will provide a base for detection of STI trends in Germany. In addition, information about sexual risk behaviour will enable us to target prevention at those most at risk for STls.
\end{abstract}

W ith the introduction of a new infection protection act in Germany in January 2001, notification for sexually transmitted infections (STIs) such as gonorrhoea, lymphogranuloma venerum, and granuloma inguinale was discontinued. Only syphilis and HIV are reported anonymously by laboratories and physicians to the Robert Koch-Institut. ${ }^{1}{ }^{2}$ Although the reported incidence of syphilis in Germany was low in the 1990s, an increase in notifications was noted after 2001 and the incidence of syphilis more than doubled between 2000 and 2002. ${ }^{3}$ The rise in syphilis cases is predominantly in men in large cities. An upsurge of reported STIs not only for syphilis but also for Chlamydia trachomatis and Neisseria gonorrhoeae has been reported over the past few years from France, United Kingdom, the Netherlands, Denmark, and Sweden, ${ }^{4-9}$ as well as eastern European countries. ${ }^{10-12}$ The increase of syphilis has been predominantly attributed to outbreaks among men who have sex with men (MSM) and a substantial proportion of these MSM have also been found to be HIV positive, ${ }^{4671314}$ raising fears that STIs are assuming an increasingly important role in the transmission of HIV. ${ }^{15} 16$ Furthermore, the number of gonorrhoea strains resistant to penicillin and ciprofloxacin has increased in France, Denmark, the Netherlands, and United Kingdom. ${ }^{5}{ }^{17-20}$ In Germany, only anecdotal evidence was available for these STIs except syphilis.

The purpose for setting up a sentinel surveillance system was to establish a surveillance for other STIs than syphilis in Germany. The objectives of the system were to assess the nation-wide occurrence of STIs and detect epidemiological trends in a timely fashion. STI patients detected by the sentinel system should be described to identify risk groups and risk behaviour. It is planned that the results produced by the sentinel system are widely disseminated and used to guide further prevention strategies.

\section{METHODS}

\section{Population under surveillance}

The population under surveillance is the population of Germany aged over 10 years living in urban and semiurban areas.

\section{Reporting sources}

In Germany, patients with STIs are mostly diagnosed by private practitioners-namely, specialists in dermatology, gynaecology, urology, or HIV. Many local health offices (LHOs) in German cities offer free STI tests and care for people without health insurance (that is, illegal migrants or sex workers). The majority of LHOs also offer anonymous HIV counselling and testing. In addition, some hospital based STI clinics provide ambulatory care for STI patients. Therefore, in order to gain comprehensive data, we wanted to include information from local health offices, hospital based STI clinics and private practitioners throughout Germany (fig 1). Neither the total number of LHOs offering HIV/STI counselling nor the number of hospital based STI clinics or HIV specialists in Germany is known. After the abolition of mandatory testing in female sex workers in 2001, the number of LHO clients dropped sharply. Consequently, an unknown number of LHOs who had put more weight on mandatory testing than on counselling in the past, disestablished HIV/STI counselling. As STI counselling and testing tends to be offered only in large cities, we chose to contact LHOs situated in cities with $>100000$ inhabitants after obtaining a formal authorisation from each federal state. The numbers of hospital based STI clinics are also unknown, as they are usually embedded in HIV or dermatology services.

Abbreviations: $\mathrm{LHO}$, local health offices; MSM, men who have sex with men; PID, pelvic inflammatory disease; STls, sexually transmitted infections 


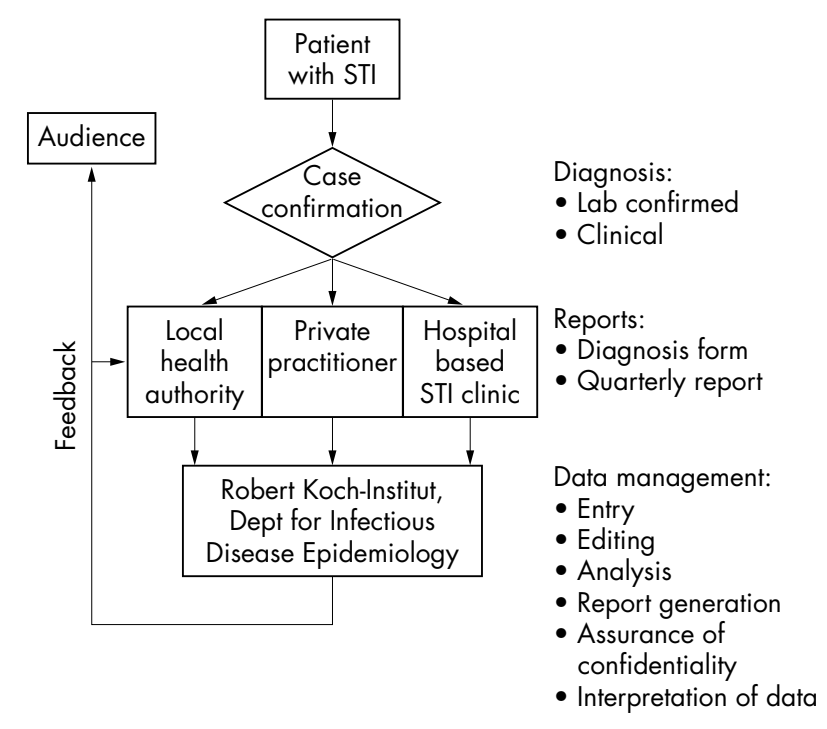

Figure 1 Flow chart for the German STI sentinel surveillance system.

Therefore, we recruited a convenience sample of those hospital based STI clinics already known to us. A total of 10139 gynaecologists, 3544 dermato-venerologists, and 2650 urologists were providing ambulatory care in Germany in 2003, but only an unknown number are involved in treating STIs on a regular base. Being an HIV specialist is not a recognised medical specialty, therefore the number of HIV specialist was unknown as well. HIV specialists were recruited with the help of a professional body which does not include all German HIV specialists.

Private practitioners were chosen randomly in a three stage stratified sample. Firstly, the number of private practitioners per federal state was chosen proportionately to population size in the federal state. Secondly, as STIs are more likely to be found in an urban setting, we aimed to select $30 \%$ of the private practitioners from a city $>500000$ inhabitants (including Berlin, Munich, Hamburg, Cologne, Frankfurt, Bremen, and Stuttgart), $50 \%$ in middle sized cities of $>100000$ inhabitants and $20 \%$ in semiurban areas. Thirdly, as STIs are more likely to be diagnosed by specialists in dermato-venerology, we aimed for $40 \%$ of the private practitioners to be dermato-venerologists, and 20\% gynaecologists, urologists, and HIV specialists, respectively. The goal was to recruit approximately 50 local health offices, 200 private practitioners and 10 hospital based STI clinics for the sentinel. From December 2002 to March 2003, we contacted 1222 private practitioners, 20 hospital based clinics and 81 LHOs by letter. Those physicians who did not spontaneously agree to participate were contacted by phone.

Owing to limited resources we were unable to compensate sentinel physicians for the extra amount of work created by their participation. Also, paying money for reporting diseases may generate similar expectations for other studies or routine surveillance. Therefore, we were aiming to use non-financial incentives for the sentinel. All institutions were given a certificate of participation on glossy paper and a free subscription of the weekly "Epidemiologisches Bulletin" published by the Robert Koch-Institut which covers work and news on infectious disease epidemiology in Germany. All participating physicians were invited to a 1 day meeting in Berlin where aims, methods and first results of the sentinel system were presented. This meeting provided an opportunity to get to know each other, discuss methodological issues, and create a network of physicians dedicated to STIs. The network effect was reinforced by six regional meetings during 2003-4.

\section{Diseases included}

According to the case definitions made available to the sentinel sites, a reportable case was defined as an individual with a laboratory confirmed episode of one of the following STIs: chlamydia, gonorrhoea, syphilis, trichomoniasis, and hepatitis $\mathrm{A} / \mathrm{B}$ (only if sexually transmitted) or a newly diagnosed HIV infection identified through routine laboratory testing. As no case definition was used for the old notification system, they could not be taken into account. The case definition for syphilis and HIV corresponds to the definition used in the new notification system. In addition, the case definition for reportable non-specific urethritis, pelvic inflammatory disease (PID), anogenital warts, and genital herpes was based on the clinical diagnosis.

The following case definitions for clinical diagnosis were made available to the sentinel sites:

- Urethritis of unknown cause: inflammation of the urinary passage with painful or burning urination, pollakiuria, urgency, discharge from the urethra without laboratory diagnosis of a pathogen.

- Genital herpes: presence of anogenital ulcerations as a new diagnosis or a relapse.

- Anogenital warts (human papillomavirus): presence of macroscopic visible genital warts on inner and outer genitals, in the perineal or perianal region, cytological evidence of koilocytosis, colposcopic mosaic, leucoplakia, intraepithelial lesions on vulva, vagina or cervix, or glans penis as a new diagnosis or a relapse.

\section{Data collected}

Physicians who agreed to participate were asked to fill in an initial questionnaire which was sent out with the recruitment letter. In this questionnaire we assessed the characteristics of the sites-namely, the size, resources, and type of patients. Sentinel physicians were asked to specify the proportion of immigrants (including illegal immigrants) and risk groups (MSM, heterosexual men or women, injecting drug users, female sex workers, or others) among their patients.

For each newly diagnosed STI patient, physicians were asked to fill in a short standardised questionnaire which was developed in collaboration with physicians dealing with STIs including the date of consultation, age, sex, residence (first three letters of zip code), country of origin, presumed route of transmission (MSM, sex work, heterosexual contacts, etc), drug habits, STI history, and HIV serostatus (diagnosis form). Next, the physician hands out a questionnaire and an information letter explaining the aims of the sentinel system to the patient (patient form). The patient form includes questions about the reason of visit to the clinic, the country of infection, the likely route and source of transmission (man/woman, steady or casual partner), drug habits, sexual behaviour (partnership, gender, and number of sex partners, commercial sex, condom use) during the past 6 months and social background (school education, financial situation, nationality, country of birth, and migration status). Patients are asked to answer the questionnaire confidentially and anonymously and forward it to the study centre by separate stamped envelope. A unique identifier number including the three digit sentinel site code and a number assigned to the sentinel site is used by the reporting physician on the diagnosis and the patient form. At the Robert Koch-Institut the patient form is matched with the diagnosis form using the unique identifier number. Furthermore, the sentinel sites were asked to report their cumulative number of attendees, the number of diagnostic tests, and positive diagnosis for each STI as well as data about genital warts and herpes 
genitalis on a monthly (for large sites) or quarterly base, including zero reporting.

Feedback on results and information on STI related topics are provided to the participants by a quarterly publication called "STD-Telegramm." Recent results are also available on the internet for the participants and have been published in the weekly bulletin ${ }^{21}$. Furthermore, methods and results of the sentinel system have been presented on national and international meetings.

\section{Data analysis}

Based on the information gained from the initial questionnaire, we compared the characteristics of the sentinel sites in relation to catchment area, number of patients, number and type of staff, the type of patients, and the proportion of immigrants attending the site.

Before the diagnosis and patient forms are entered into the database, a quality and plausibility check is performed. If items are missing or demographic information on the diagnosis form is differing from that in the patient form, physicians are contacted to obtain complete information. Similarly, if the number of received diagnosis forms differs from the declared number of diagnosed STIs on the monthly/ quarterly form, physicians are asked to complete the remaining forms if possible.

Data are analysed regularly to detect changes in the number of reported STIs in relation to the number of sentinel

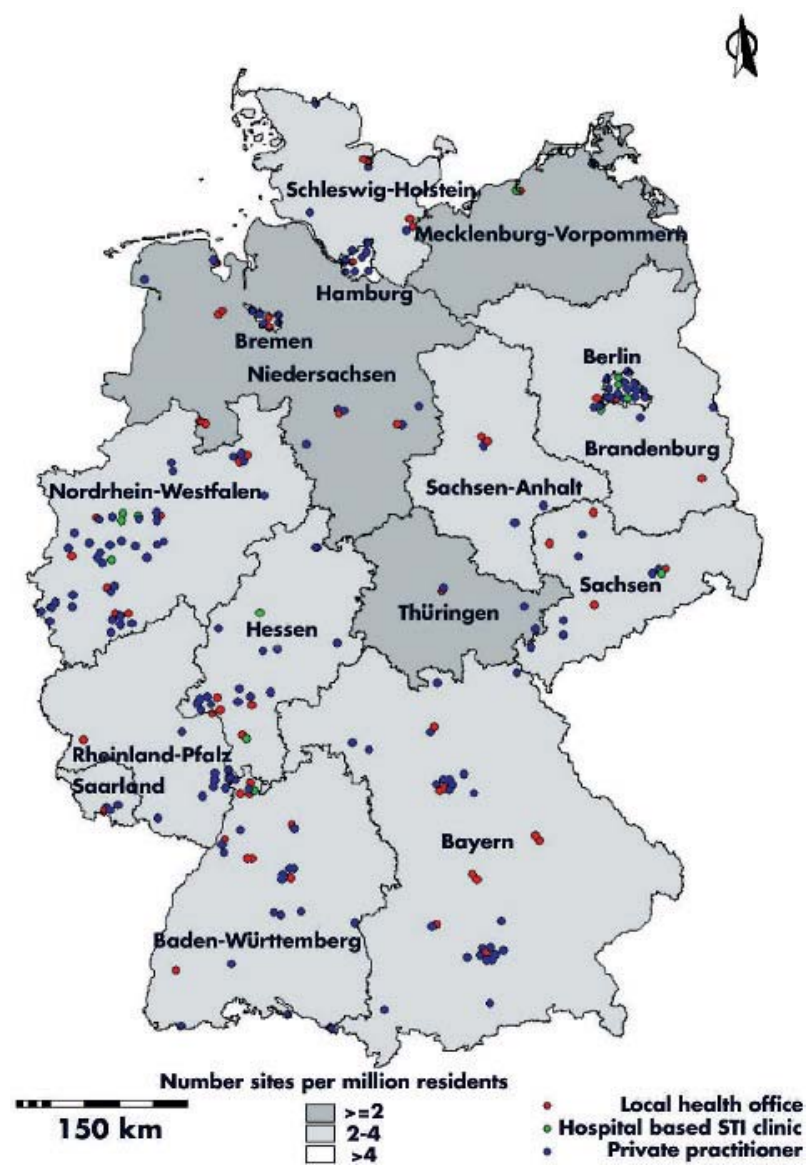

Figure 2 Location of sentinel sites in Germany and number of sentinel sites per million residents in German federal states, September 2003. Red dots are local health offices, green dots are hospital based STI clinics, and blue dots are private practitioners. High number of sentinel sites per million residents are shown in white, medium and low number of sentinel sites in light and dark grey, respectively. The federal states of Berlin, Hamburg, and Bremen have $>4$ sites/million residents. sites, number of attendees seeking STI care per sentinel site, and number of diagnostic tests. For each type of STI we determine demographic characteristics, geographic distribution, frequency of STI history, and the likely source of infection of the patients. Using the information from the patient forms we try to identify sexual risk behaviour patterns specific for each disease. Differences between STIs are calculated using the $\chi^{2}$ test and Student's $t$ test. Subgroups of patients like MSM, female sex workers, patients aged under 25 years (and not belonging to the previous two groups), and patients with a migration background are analysed separately. By assembling data from the sentinel sites over a certain period of time, the analysis of temporal and geographical clusters of STIs as well as trends will be carried through.

In order to assess the sensitivity of the sentinel system according to the CDC guidelines for evaluating public health surveillance systems, ${ }^{22}$ characteristics of syphilis and HIV patients reported through the sentinel were compared to those reported by the national notification system during the same time period.

\section{Data protection}

As STIs are a sensitive issue, great care was taken regarding confidentiality of the collected data. The German law on data protection requires that data should be anonymised to make them virtually impossible to draw conclusions from a reported case to an individual person. Therefore, only a three digit postal code could be used and data on country of origin are analysed in groups. In order to match the diagnosis form with the patient form, a reference number including the three digit sentinel site code is used by the reporting physician on the diagnosis and the patient form. This number, which is the only information referring to the reporting site, is removed after matching. Filling out the patient form is voluntary and anonymous and should be done without the interference of health professionals. The patient forms were translated into the most commonly used languages among foreign nationals (French, English, Spanish, Russian, Polish, Turkish, Thai, Bulgarian, and Romanian) to allow patients with little knowledge of German to fill in the forms autonomously.

The data set is protected by personal passwords and accessible to only five people directly involved with the surveillance system.

\section{Resources}

Funding for a full time employed information specialist responsible for data entry, checking for plausibility, and administrative work was provided by the German Ministry of Health as well as printing and translation of the forms, travel and the sentinel participants meeting for a length of 3 years. All other costs (computer hardware, postal and telephone charges) are covered by the Robert Koch-Institut. The need to continue the sentinel system will be reassessed before the end of the funding period.

\section{RESULTS}

\section{Acceptability}

Between December 2002 and March 2003 we recruited 58 LHOs, 14 hospital based clinics, and 160 private practitioners in 112 cities. Participating institutions are present in all federal states; however, there is a concentration on densely populated urban areas like Berlin, Hamburg, Munich, and the Ruhr basin (fig 2); 54 (33.8\%) of the private practitioners are situated in cities with $>500000$ inhabitants and a further 56 $(35.0 \%)$ in cities with $>100000$ inhabitants. A total of 176 of $232(75.9 \%)$ sentinel sites was located in cities with $>100000$ inhabitants. 


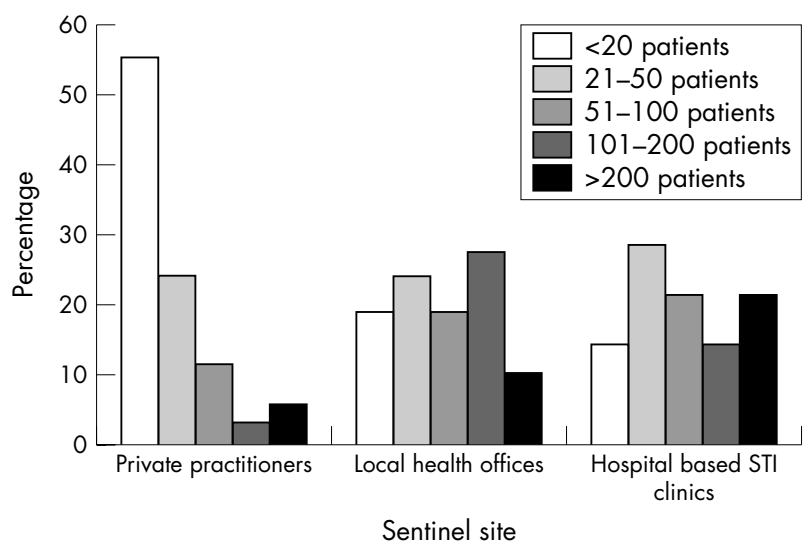

Figure 3 Number of STI patients treated by sentinel sites per month (local health office, hospital based STI clinic) or quarter (private practitioners).

In all, 58 LHOs with STI and/or HIV care/management from 44 cities are part of the sentinel network. Twelve $(20.7 \%)$ of these sentinel sites are located in cities with $>500000$ inhabitants. Eighteen of the LHOs offer HIV testing and counselling, 20 care/management of STI, the remaining 20 joint HIV and STI care/management. Of the 160 private practitioners, $85(53.1 \%)$ are dermato-venerologists, 30 $(18.8 \%)$ gynaecologists, 25 (15.6\%) urologists, and 20 $(12.5 \%)$ HIV specialists. Forty six $(28.8 \%)$ of the sentinel physicians work in group practice with a median of two colleagues. Of the participating 14 hospital based clinics, nine were university hospitals, four communal hospitals, and one a teaching hospital. Of the 232 sentinel sites, 32 (13.8\%) did not send any report during the first year of the sentinel.

\section{Patients attending the sentinel sites}

The number of attendees seeking STI care (including HIV testing) per month (LHOs and hospital based STI clinics) or per quarter (private practitioners) are shown in figure 3. While $55 \%$ of the private practitioners reported caring for up to twenty persons seeking STI care per quarter, over $80 \%$ of LHOs and hospital based clinics reported taking care of more than 20 people seeking STI care per month. Local health offices had the highest median proportion of women (60\%) and legal as well as illegal immigrants among their clientele. The median proportion of immigrants among their attendees was $10 \%$ for private practitioners, $22.5 \%$ for LHOs, and $20 \%$ for hospital based clinics. When asked about the type of male patients, heterosexual men were mainly cared for by private practitioners and LHOs (median proportion 45\% and $78.5 \%$ respectively), whereas the median proportion of MSM was the highest in hospital based STI clinics (50\%). Only LHOs reported a significant proportion (47.5\%) of sex workers among their female STI patients. A small proportion $(5 \%)$ of injecting drug users among STI attendees were reported from hospital based clinics. According to the information provided by the patients, almost half $(48.7 \%)$ of the patients visited a physician because they had symptoms, $28 \%$ wanted to have a check up, $7 \%$ came because their partner had an STI.

From l March 2003 to February 29, 2004, a total of 2071 cases of STIs in 1833 patients were reported to the Robert Koch-Institut. The number of monthly reported STIs remained stable with a median of 158 cases per month (fig 4). Among the reported STIs were 452 cases of chlamydia, 343 gonorrhoea, 321 syphilis, $269 \mathrm{HIV}$, and 90 trichomoniasis. The proportion of men as well as coinfections with syphilis or chlamydia or HIV are shown in table 1 . In addition we received 41 reports on hepatitis $\mathrm{A}, 78$ on hepatitis B, 123 on non-specific urethritis, 130 on pelvic inflammatory disease, and 224 other STIs; 925 (50.5\%) of the patients were male, the median age was 31 years (range 1480 years). Female STI patients were more often of foreign origin than men $\left(\chi^{2}\right.$ test; $70.0 \% v 26.3 \%$; $\left.<<0.001\right)$.

Of these 1833 STI patients, we received 651 patient forms, which corresponds to a response rate of $35.5 \%$. Of the respondents, $380(58.4 \%)$ were male. Non-responders were younger than responders ( $\chi^{2}$ test; median age 30 years $v$ 33 years; $p<0.001)$. Significantly more German than nonGerman STI patients sent back the questionnaire $\left(\chi^{2}\right.$ test; $64.8 \%$ v 26.1\%; $\mathrm{p}<0.001)$. Among the respondents we identified 279 (42.9\%) MSM, 197 (30.3\%) female sex workers, $44(8.1 \%)$ aged under 25 years, and 240 (36.9\%) migrants.

Of the 947 received cumulative quarterly and monthly forms that covered the period between March 2003 and February 2004, the median number of STI patients cared for per month was 71 in LHOs and 30 in hospital based clinics. Private practitioners were seeing a median of eight STI patients per quarter (Kruskal-Wallis test; $\mathrm{p}<0.001$ ). The median total number of treated patients per quarter was 19377 (range 18 988-19 916).

\section{Data quality}

Of the data entered in the database, 20 (1.1\%) of 1833 diagnosis forms had no information on age, 45 (2.5\%) did not mention the country of origin and route of transmission was

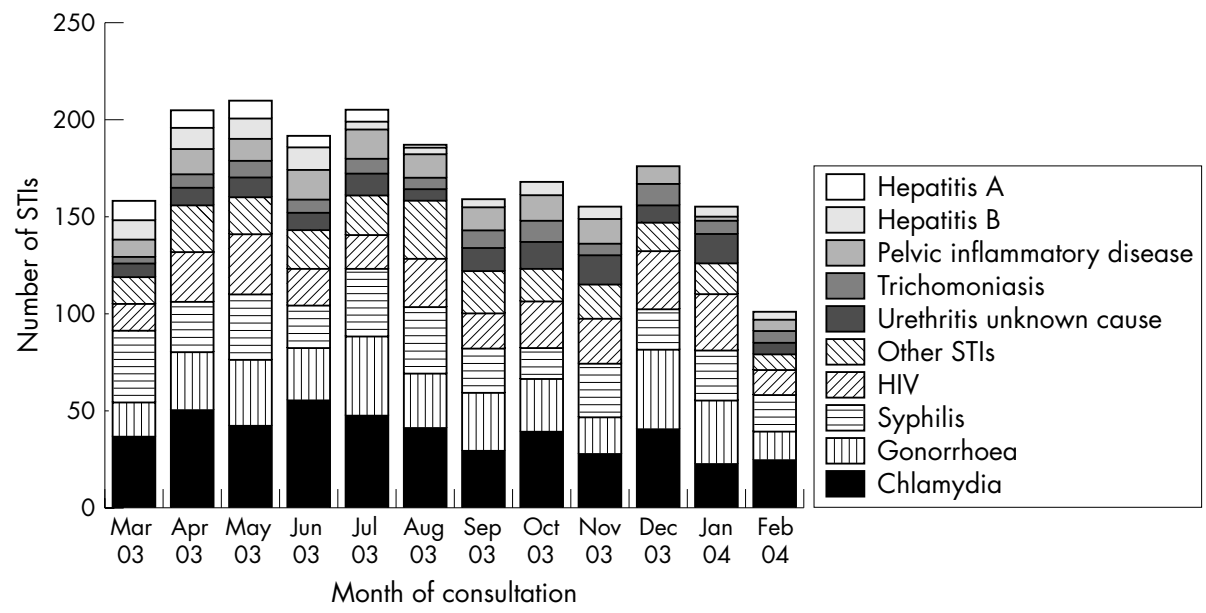

Figure 4 Number of diagnosed STIs per month of consultation, March 2003 to February 2004 as of 10 May 2004. 
Table 1 Number of reported cases, proportion of male patients, median age in years, number and proportion of patients of non-German origin for chlamydia, syphilis, gonorrhoea, HIV, and trichomonas as well as concurrent diagnosed co-infections with HIV, syphilis, or chlamydia from March 2003 to February 2004 as of 10 May 2004

\begin{tabular}{|c|c|c|c|c|c|c|c|c|}
\hline \multirow[b]{2}{*}{ Diagnosis } & \multirow[b]{2}{*}{$\begin{array}{l}\text { Number of } \\
\text { casest }\end{array}$} & \multicolumn{2}{|c|}{ Demographic characteristics } & \multicolumn{2}{|l|}{ Risk group } & \multicolumn{3}{|c|}{ Co-infection with } \\
\hline & & $\begin{array}{l}\text { Male patients } \\
(\%)\end{array}$ & $\begin{array}{l}\text { Median age } \\
\text { (years) }\end{array}$ & $\begin{array}{l}\text { Patients of non- } \\
\text { German origin } \\
\text { (\%) }\end{array}$ & MSM (\%) & $\begin{array}{l}\text { Chlamydia } \\
(\%)\end{array}$ & Syphilis (\%) & HIV (\%)§ \\
\hline Chlamydia & 452 & $144\left(31.9^{*}\right)$ & 26 & $239(52.9)$ & 51 (11.3) & - & $12(2.7)$ & $21(4.6)$ \\
\hline Syphilis & 321 & $270\left(84.1^{*}\right)$ & 36 & $87(27.1)$ & $213(66.4)$ & $12(3.7)$ & - & $127(28.6)$ \\
\hline HIV & 269 & $234\left(87.0^{*}\right)$ & 34 & $88(32.7)$ & $174(64.7)$ & 5 (1.9) & $34(12.6)$ & - \\
\hline Gonorrhoea & 343 & $203\left(59.2^{*}\right)$ & 30 & $158(46.1)$ & 115 (33.5) & 37 (10.8) & $9(2.6)$ & 54 (15.7) \\
\hline Trichomonas & 90 & $3\left(3.3^{*}\right)$ & 28 & $61(67.8)$ & 0 & $5(5.6)$ & $1(1.1)$ & $2(2.2)$ \\
\hline
\end{tabular}

missing in $73(4.0 \%)$ forms. Information on drug habits and STI history was missing in $726(39.7 \%)$ and $267(14.6 \%)$, respectively.

\section{Sensitivity}

From l March 2003 to 29 February 2004, 1710 newly diagnosed HIV infections and 2950 syphilis cases have been notified through the new statutory reporting system. If we assume that laboratory notifications for HIV and syphilis are complete, the coverage of the sentinel system account for $15.7 \%$ and $10.9 \%$ of HIV and syphilis in Germany. The proportion of male patients in the sentinel was higher for HIV (87.0\% $v 78.9 \%)$, but lower for syphilis (84.1\% $v 90.2 \%)$. Median age was similar for HIV (33v 34 years) and syphilis patients (36 years) in sentinel and notification patients. In both systems, most infections were reported from the federal states of Berlin, Bavaria, Baden-Württemberg, North RhineWestphalia, Hamburg, and Saxony.

\section{Timeliness}

In all, $27.7 \%$ of the reports were sent in during the same quarter and $64.1 \%$ during the following quarter. The mean time lag between the date of consultation and data entry was 76 days.

\section{DISCUSSION}

As neighbouring countries reported increasing numbers of STIs and data were scarce in Germany, our aim was to set up a surveillance system for STIs. Since STIs were presumably under-reported by former routine notification, ${ }^{23}$ we believed that a sentinel system involving motivated physicians would improve the quality of the reports. ${ }^{2}$ Furthermore, a sentinel system provides a frame for collecting more detailed information which can be a useful guide to target intervention strategies. ${ }^{24}$

The German STI sentinel system was conceived to gain data on STIs from different risk groups as well as the general population. Thus, unlike the STI sentinel system in Italy, ${ }^{25}$ different healthcare providers were included in the surveillance system. If we had merely recruited LHOs, we would not have had enough information on STIs among heterosexual men and women. However, the number of STI patients cared for by private practitioners reportedly was significantly smaller than in other sentinel sites. Sentinel sites were recruited in all federal states proportional to the population size. This was important for a country like Germany where considerable social and economic differences between federal states prevail. Still, the majority of the sentinel sites are located in middle sized to large cities, where we can expect to detect STI trends earlier than in a rural setting.

\section{Methodological issues}

The German sentinel surveillance system, nevertheless, has a few limitations: we have recruited a relatively small number of sentinel sites. This was mainly because of a low response rate among private practitioners, a phenomenon previously observed in Germany and France. ${ }^{26}{ }^{27}$ The system will allow us to detect outbreaks in larger cities with several sentinel sites. Smaller outbreaks or outbreaks in smaller cities might go unnoticed by this system. The sentinel system cannot be used to extrapolate prevalence or incidence of STIs from the results as (a) sentinel sites are probably not representative of all STI provider in Germany; (b) owing to the free choice of medical practitioners, the catchment areas of the sites cannot be determined. A comparison of sentinel data with other national surveillance systems is therefore not feasible.

Regarding the accuracy of the system, many patients are asymptomatic, STI symptoms are not recognised, or physicians omit laboratory testing for financial reasons. This can lead to an underestimation of the prevalence of STIs in patients attending the services which are part of the sentinel.

\section{Simplicity and flexibility}

We aimed to keep the system as simple as possible by accepting routine laboratory testing. Data on STI patients are forwarded directly to the Robert Koch-Institut. Physicians only need to supply a restricted amount of information as most information on sexual behaviour is collected directly from patients. In addition, only one person is needed to maintain the quality of the system. Since the number of sentinel sites is rather small, changes in the system could be implemented easily if necessary.

\section{Data quality and acceptability}

The results of the sentinel showed that completeness of essential data like demographic information was high. As data quality is regularly checked and improved, we believe that data quality of the STI sentinel is higher than in a statutory notification system.

It has been shown on several occasions that self reported sexual behaviour is quite reliable, but the quality can vary considerably. ${ }^{28}{ }^{29}$ Social desirability might influence information on number of sexual partners or condom use. ${ }^{28}$ Even if this effect was considerable, we believe that we can gain valuable first hand information from STI patients on the possible source of infection and risk behaviour such as drug use, number of partners, condom use, and commercial sex. This will enable us to identify a sexual behaviour pattern for people seeking treatment in the sentinel sites, especially in the absence of survey data on symptomatic and asymptomatic STI patients. However, we have to keep in mind that 


\section{Key messages}

- Surveillance for STIs is needed in Germany, therefore a sentinel surveillance system has been built

- Despite some limitations, the STI sentinel surveillance system provides a fairly good overview of the distribution of STls and the characteristics of STI patients in Germany

- Additional information on sexual behaviour will help to guide targeted intervention strategies

sexual risk behaviour of the STI patients may not reflect sexual risk behaviour in the community. ${ }^{24}$ In addition, data on sexual behaviour of STI patients with a migration background may not be representative of all immigrants with STIs. Despite translated questionnaires being provided to the sentinel sites, we believe that the lack of questionnaires in their mother tongue, poor reading and writing skills, the illegal status of many patients and a lack of confidence towards authorities contribute to the significantly lower response rate in this group.

Although it was difficult to recruit private practitioners, most participating sites send information on a regular basis, showing that the acceptability of the system among the participants is high.

\section{Sensitivity}

The sensitivity of the sentinel system can be estimated by comparing the reported cases of HIV and syphilis to the statutory notification system. Despite the small number of sentinel sites, the coverage for HIV as well as for syphilis exceeds $10 \%$. This has to be reassessed regularly to ensure that the quality of the sentinel system is maintained. Whether the system is sensitive enough to detect outbreaks remains to be shown.

\section{Positive predictive value and representativeness}

The positive predictive value cannot be assessed at this stage as other sources-that is, medical records, have not been reviewed to confirm cases. The comparison between notified HIV and syphilis cases and cases reported through the sentinel system has shown that demographic characteristics are similar in many respects. Hence, we can believe that other STIs reported by the sentinel system are somewhat representative of the whole population of STI patients.

\section{Timeliness and stability}

Another concern is the timeliness of the reports. We have to put more effort into reducing the time lag between the diagnosis and the reports. This could be done by extending the possibility of electronic reporting. Since the sentinel system was set up, it remained stable in terms of participants.

Our sentinel system will provide a base for detection of STI trends in Germany. STI surveillance is not only important on a national, but also on an international, level as cooperation in infectious disease surveillance between European countries is growing. ${ }^{30}$ Provided high data quality are kept, the sentinel is a tool that will enable us to target prevention at those most at risk from STIs.

\section{ACKNOWLEDGEMENTS}

We thank Michael Kramer from the German Ministry of Health and Social Security for his constructive comments when planning the sentinel system. We are deeply indebted to our colleagues Dorothea Matysiak-Klose, Sabine Ritter, and Johanna Puscher for helping us in recruiting private practitioners for the sentinel, as well as Bernd Reinhardt who has set up the database. We would like to thank all the staff of the participating sentinel sites for taking extra time to complete the different forms and handing out the patient forms to their patients. Finally, we are grateful to all the patients who were willing to provide us with extra information regarding their sexual behaviour.

\section{CONTRIBUTORS}

VB is responsible for the implementation of the study, she drew up the study protocol, recruited the participants, performed the statistical analysis, and wrote the manuscript; UM has participated in the design of the questionnaires; $\mathrm{AH}$ recruited the participants, helped create the database, performed the data entry, and dealt with data quality issues; $\mathrm{OH}$ is responsible for the Unit for HIV/AIDS/STI within the Department of Infectious Diseases Epidemiology and is project leader, he gave his advice on the study protocol, assured the funding for the study, and supervised the study.

\section{Authors' affiliations}

V Bremer, U Marcus, A Hofmann, O Hamouda, Department for Infectious Disease Epidemiology, Robert Koch-Institut, Berlin, Germany

Sources of funding: The project is funded by the German Ministry of Health.

Conflict of interest: None.

\section{REFERENCES}

1 Robert Koch-Institut. Umsetzung der Meldung nach §7 Abs. 3 des Infektionsschutzgesetzes. Bundesgesundheitsblatt-Gesundheitsforschung Gesundheitsschutz 2000;43:875-9.

2 Petzold D, Jappe U, Hartmann M, et al. Sexually transmitted diseases in Germany. Int J STD AIDS 2002;13:246-53.

3 Robert Koch-Institut. Jahresbericht Syphilis. Epidemiologisches Bulletin 2003:35:277-9.

4 Nicoll A, Hamers FF. Are trends in HIV, gonorrhoea, and syphilis worsening in western Europe? BMJ 2002;324:1324-7.

5 Goulet V, Sednaoui P, Laporte A, et al. The number of gonococcal infections identified by the RENAGO network is increasing. Euro Surveill 2000:5:2-5.

6 Crook PD, Paine TC, Davis M, et al. London-the next battleground for syphilis? Commun Dis Public Health 2002;5:163-4.

7 Axelsen N, Smith E, Kock-Hansen GH. Syphilis cases increasing in Denmark, 2000-2001. Eurosurveillance Weekly 2002;6:6-8.

8 Berglund T, Fredlund $\mathrm{H}$, Giesecke J. Epidemiology of the reemergence of gonorrhea in Sweden. Sex Transm Dis 2001;28:111-14.

9 Berglund T, Blystad H. Increase in genital chlamydia infections in Sweden. Eurosurveillance Weekly 2002;6(26).

10 Borisenko KK, Tichonova LI, Renton AM. Syphilis and other sexually transmitted infections in the Russian federation. Int J STD AIDS 1999;10:665-8.

11 Gomberg MA, Vagan AA. Resurgence of sexually transmitted diseases in Russia and eastern Europe. Sex Transm Dis 1998; 16:659-62.

12 Mavrov GI, Bondarenko GM. The evolution of sexually transmitted infections in the Ukraine. Sex Transm Infect 2002;78:219-21.

13 Lynch A, Smyth B. Syphilis outbreak in Northern Ireland. Eurosurveillance Weekly 2003;7:5-8.

14 Vilayleck $M$. Continuing resurgence of syphilis in France. Eurosurveillance Weekly 2001;5:1-2.

15 Halsos AM, Edgardh K. An outbreak of syphilis in Oslo. Int J STD AIDS 2002;13:370-2.

16 Lowndes C. Sexually transmitted infections may assume increasingly important role in the evolution of HIV transmission in Europe. Eurosurveillance Weekly 2002;6:6-7.

17 Hoffmann S. The laboratory surveillance system of Chlamydia trachomatis and Neisseria gonorrhoeae infections in Denmark. Eurosurveillance $2001 ; 6: 86-90$.

18 Fenton KA, Ison C, Johnson AP, Rudd E, Soltani M, Martin I, Nichols T, Livermore DM on behalf of the GRASP collaboration. Ciprofloxacin resistance in Neisseria gonorrhoeae in England and Wales in 2002. Lancet 2003;361:1867-9.

19 Peerbooms PG, Spaargaren J, Fennema JS, et al. [Increased Neisseria gonorrhoeae quinolone resistance in Amsterdam]. Ned Tijdschr Geneeskd 2001;145: 1899-900.

20 Rudd E, Fenton K. Large increase in ciprofloxacin resistant gonorrhoea in England and Wales. Eurosurveillance Weekly 2003;16:9-10.

21 Bremer V. (The STI sentinel system of the Robert Koch-Institut-first results.) Epidemiologisches Bulletin 2004:1.

22 Centers for Disease Control. Updated guidelines for evaluating public health systems. Recommendations from the guidelines working group. MMWR 2001;50:RR-13. 
23 Kirschner W, Schwartländer B. Sentinel Surveillance von HIV und anderen sexuell übertragbaren Krankheiten-Ergebnisse der ANOMO-Studie 1988-1994. Hrsg Das Bundesministerium für Gesundheit. Baden-Baden, Nomos verl-Ges, 1996.

24 Howards PP, Thomas JC, Earp JA. Do clinic-based STD data reflect community patterns? Int J STD AIDS 2002;13:775-80.

25 Dal Conte I, Lucchini A, Contuzzi E, et al. Sexually transmitted infections in Italy: an overview. Int J STD AIDS 2001;12: 813-18

26 Szecsnyi J, Uphoff $\mathrm{H}$, Ley $\mathrm{S}$, et al. Influenza surveillance: experiences from establishing a sentinel surveillance system in Germany. J Epidemiol Community Health 1995;49(Suppl 1):9-13.
27 Hanslik T, Boelle PY, Flahault A. Setting up a specific surveillance system of community health during mass gatherings. J Epidemiol Community Health 2001;55:683-4.

28 Morisky DE, Ang A, Sneed CD. Validating the effects of social desirability on self-reported condom use behavior among commercial sex workers. AIDS Educ Prev 2002;14:351-60.

29 Durant LE, Carey MP. Reliability of retrospective self-reports of sexual and nonsexual health behaviors among women. I Sex Marital Ther 2002;28:331-8.

30 Fenton A, Giesecke J, Hamers FF. Europe-wide surveillance for sexually transmitted infections: a timely and appropriate intervention. Euro Surveill $2001 ; 6: 69-70$. 